\title{
Impact of additional fructo-oligosaccharides on the gastrointestinal microbiota, fermentation and stool output in patients receiving enteral nutrition on the intensive care unit: a multi-centre, randomised, double-blind, controlled trial
}

\author{
H. A. Majid ${ }^{1}$, J. Cole ${ }^{1}$, C. L. Reid ${ }^{2}$, T. Sherry ${ }^{2}$, R. J. Beale ${ }^{2}$, M. Ervine ${ }^{3}$, \\ P. W. Emery ${ }^{1}$ and K. Whelan ${ }^{1}$ \\ ${ }^{1}$ King's College London, Diabetes and Nutritional Sciences Division, London, SE1 9NH, UK, ${ }^{2}$ Intensive Care Unit, Guy's \\ and St Thomas' NHS Foundation Trust, London, SE1 7EH and ${ }^{3}$ Intensive Care Unit, King's College Hospital NHS \\ Foundation Trust, London, SE5 9RS, UK
}

The gastrointestinal (GI) microbiota produce short-chain fatty acids (SCFA) that stimulate colonic water absorption and may protect against enteropathogenic colonisation (e.g. Clostridium difficile). However, alterations in the GI microbiota can occur during enteral nutrition (EN), with patients who develop diarrhoea having lower faecal concentrations of bifidobacteria ${ }^{(1)}$. Prebiotic fructooligosaccharides (FOS) stimulate the growth of bifidobacteria in healthy people. However, in patients receiving EN, no differences in bifidobacteria were found between those receiving standard formulas or those containing fibre and FOS at the levels found in current formulations $^{(2)}$. Therefore, the aim of this study was to investigate the effect of additional FOS supplementation on the GI microbiota, SCFA and stool output in patients receiving EN on the intensive care unit (ICU).

Adult patients on the ICU who were starting EN with a formula containing fibre and FOS were eligible for inclusion. Patients with a GI disorder or prescribed lactulose were excluded. Patients were randomised to receive $7 \mathrm{~g} / \mathrm{d}$ of additional FOS or an identically packaged non-prebiotic placebo (maltodextrin) for 12-14 days. A fresh stool sample was collected at baseline and following 12-14 days of EN and additional FOS/placebo. Faecal microbiota were analysed using fluorescent in situ hybridisation using oligonucleotide probes targeting bifidobacteria, lactobacilli, clostridia, bacteroides and Faecalibacterium prausnitzii. Faecal SCFA were analysed using gas liquid chromatography. Stool output was monitored daily using the King's Stool Chart. Data were compared between groups using an unpaired t-test or Mann-Whitney test. The study was approved by an NHS Research Ethics Committee and assigned a clinical trials registration number (ISCRTN 06446184).

Of the 35 patients who were randomised and started the intervention, only 18 completed the full 12-14 day period ( 9 FOS, 9 placebo). There were no differences in baseline characteristics between groups. At the end of the trial, there were no significant differences in any of the microbiota between groups, although the higher concentrations of $F$. prausnitzii (median, IQR) in the placebo group (8.6, $1.3 \log _{10} / \mathrm{g}$ dry faeces) compared with the FOS group (6.0, $1.6 \log _{10} / \mathrm{g}$ dry faeces) approached significance $(\mathrm{P}=0.063)$. No patients developed C. difficile infection. There were no significant differences in any of the SCFA between the FOS and placebo groups, nor were there differences in daily stool score or incidence of diarrhea between groups.

Provision of an additional $7 \mathrm{~g} / \mathrm{d}$ FOS to patients receiving EN on the ICU does not result in beneficial changes in the microbiota, fermentation and stool output. The potential impact of FOS in lowering F. prausnitzii is of concern as this bacterium is a major butyrate producer. However, there were many confounding variables in this ICU population (e.g. variations in age, antibiotic exposure, disease), which in light of the small sample size may limit interpretation of these findings. Despite this, the current findings suggest that supplementation of fibre/FOS-containing formulas with an additional $7 \mathrm{~g} / \mathrm{d}$ FOS may be of no benefit to patients on the ICU.

Funded in part by a grant from the Nutricia Research Foundation

1. Whelan K, Judd PA, Tuohy KM, Gibson GR, Preedy VR \& Taylor MA (2009) Am J Clin Nutr 89, 240-247.

2. Majid HA, Emery PW \& Whelan K (2011) J Hum Nutr Diet 24, 260-8. 